\title{
Is discrimination enhanced at a category boundary? The case of unique red
}

\author{
M.V.Danilova, ${ }^{1,2}$ J.D.MOLLON ${ }^{2}$ \\ 1.P. Pavlov Institute of Physiology, Nab. Makarova 6, St. Petersburg 199034, RUSSIA \\ ${ }^{2}$ Department of Experimental Psychology, University of Cambridge, Downing St., CB2 3EB, UK \\ *Corresponding author: mar.danilova@gmail.com
}

Received XX Month XXXX; revised XX Month, XXXX; accepted XX Month XXXX; posted XX Month XXXX (Doc. ID XXXXX); published XX Month XXXX

Is chromatic discrimination enhanced at the boundary between different hues? In previous studies we gave a positive answer for the case of the locus of unique blues and yellows, the boundary that divides color space into reddish and greenish hues. But we did not find enhancement at the locus of unique green, the boundary between yellowish and bluish hues. In the present study we examined discrimination near the locus of unique red. In interleaved experimental runs, we obtained (i) discrimination thresholds using a four-alternative spatial forced choice and (ii) phenomenological judgments of the locus of unique red. When measurements were made along lines parallel to the locus of unique blues and yellows in a MacLeod-Boynton diagram, the locus of minimal thresholds coincided approximated with the locus of unique red; but this was not the case when measurements were made along lines orthogonal to the locus of unique blues and yellows. To account for these and earlier results, we suppose that the neural channel that determines the discrimination threshold will sometimes coincide with the channel that determines the perceptual hue equilibrium, and sometimes will not. If a given point in chromaticity space is a unique hue, then it is expected to remain a unique hue independently of the direction in which measurements are made; but discrimination thresholds almost certainly will depend on different underlying channels when measurements are made in different directions through the same point in chromaticity space. (C) 2015 Optical Society of America

\section{OCIS codes}

http://dx.doi.org/10.1364/AO.99.099999

\section{INTRODUCTION.}

Within the foveal region, normal human vision is trichromatic, depending on the presence of three classes of cone. Since the signal of each cone is univariant, representing only the total excitation of the cone [1], color discrimination depends on a comparison of the rates at which photons are being absorbed in the three classes of cone. In principle, therefore, all physical colors can be represented in terms of two ratios and so can be plotted in a two-dimensional plane. An example of such a 'chromaticity diagram' is the familiar MacLeodBoynton diagram (Fig. 1a), which takes as its axes $\mathrm{L} /(\mathrm{L}+\mathrm{M})$ and $\mathrm{S} /(\mathrm{L}+\mathrm{M})$, where $\mathrm{L}, \mathrm{M}, \mathrm{S}$ represent the excitations of the long-, middleand short-wave cones respectively $[2,3]$.

Although a chromaticity diagram represents a physically continuous space, human perception imposes on it distinct categories. The MacLeod-Boynton diagram is divided into regions of reddish and greenish hues by an oblique line that runs from unique blue to unique yellow (approximately 475 to $575 \mathrm{~nm}$, but varying with the state of adaptation); and the diagram is further divided into regions of yellowish and bluish hues by a line that runs nearly horizontally from an extra-spectral red to the white point and then more obliquely from the white point to unique green $(\sim 520 \mathrm{~nm})$ [4-9].

In the case of speech perception, it was classically found by Liberman and colleagues that discrimination was superior at the boundary between perceptual categories, e.g. at the phonemic boundaries between the voiced stops $b, d$ and $g$ or at the boundary between voiced and unvoiced stops $[10,11]$. The analogous question has often been asked in the case of color perception: Is discrimination enhanced at the boundary between different categories of hue? [1216]. Most commonly, the question has been asked about the transitions that occur between adjacent colors in a hue circle, such as red and yellow. The blue-green region of the hue circle has been especially popular, since this region is divided in different ways by different languages [17-19] However, it is also possible to ask the question about the transitions which occur at chromaticities that under a given state of adaptation - are seen as unique hues [20]. Thus a very fundamental boundary in color space is formed by the line that runs from unique blue through white to unique yellow - the boundary 
that divides the total gamut of all chromaticities into reddish and greenish hue.

If discrimination were found to be optimal at a boundary between particular subjective categories, then a mechanistic explanation would be needed. If the discriminanda were separated in time or in space, then it is possible that the comparison might be based on verbal or symbolic labels and so we might readily expect superior performance when the targets were drawn from different categories. Perhaps too we might expect category boundaries to affect the speed with which supra-threshold pairs of stimuli were differentiated. But for the case of simultaneous discrimination of near-adjacent stimuli by practiced observers, the comparison is likely to depend directly on sensory signals, that is, on the differential sensitivity of neural channels in the early visual system. This has been the almost universal assumption amongst those visual scientists who have studied color discrimination either along the spectrum locus or in different directions around a point in color space [e.g. 21-29].

Why then should we predict an enhanced resolution at a category boundary? It would be necessary to suppose either (i) that the acquisition of categories altered the sensory representation of colors at early stages of the visual system, or (ii) that color categories and color discrimination both arose from the same inbuilt property of the human visual system. An hypothesis of the latter class might postulate, say, a retinal channel that signaled redness or greenness by departures from its equilibrium state (or paired channels, one signaling redness and one signaling greenness). Since neural channels are typically most sensitive at a null point set by the current state of adaptation [30,31], we might expect such a channel to exhibit optimal resolution for chromaticities close to white or close to unique blues or unique yellows. Such a channel would not correspond to one of the two channels classically identified in the primate retina and lateral geniculate nucleus [32, 33]; but we have previously postulated a channel of this kind - drawing opposed inputs from the L and S cones on the one hand and M cones on the other - in order to explain the coincidence of minimal thresholds and phenomenal equilibria at the yellow-blue line [20,34].

However, if we consider the issue from a visual science perspective, rather than from the perspective of cognitive psychology, then it is not necessary that the same underlying channel should invariably determine both discrimination thresholds and the phenomenological equilibrium: Under some conditions the two measures may depend on different neural channels. In the case of unique green, we have previously found no indication of enhanced discrimination at the category boundary [35]. Rather thresholds were always minimal when the $\mathrm{L} /(\mathrm{L}+\mathrm{M})$ coordinate corresponded to that of the white adapting field, suggesting that the discrimination was based on one of the classical 'cardinal' chromatic channels, a channel that extracted the ratio of excitation in long- and middle-wave cones [32]. In the present paper we examine the case of unique red. When the category boundary between bluish and yellowish reds is traversed in one direction, we find that optimal discrimination does approximately coincide with the phenomenological equilibrium point; but when the category boundary is traversed in a different direction, this is not the case.
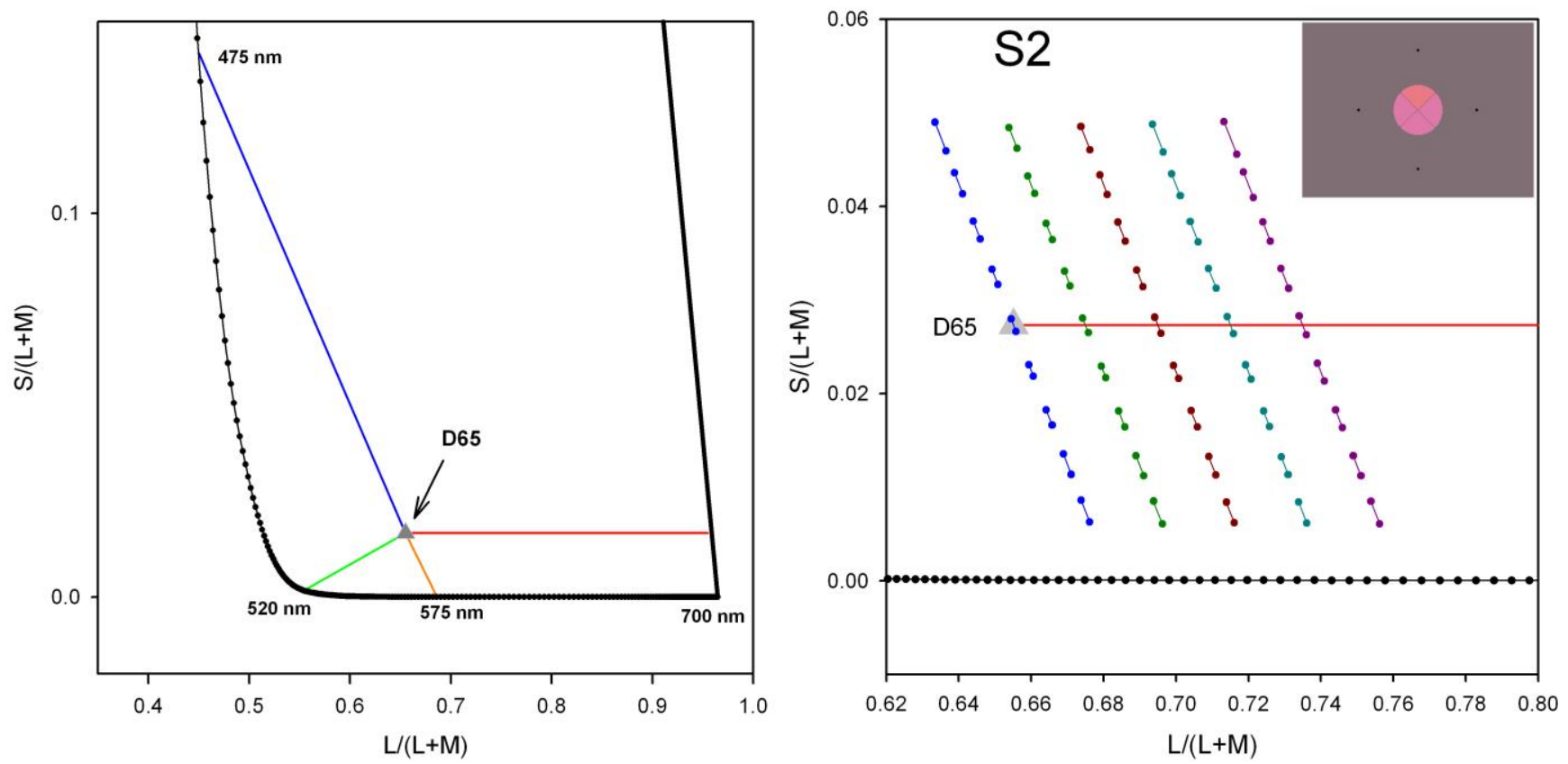

Figure 1. 1a. Part of the MacLeod-Boynton chromaticity diagram, constructed from the cone sensitivities of DeMarco, Pokorny and Smith [37]. L, M, $\mathrm{S}$ are the excitations of the long-, middle- and short-wavelength cones respectively. 'D65' indicates the chromaticity of the standard daylight illuminant D65 [51], the chromaticity used as the background in the present experiments. The solid black points represent the locus of monochromatic lights; and the solid black line represents the locus of purples. The two axes of the diagram represent ratios of cone excitations and vary continuously, but human perception imposes discontinuous hue categories on the input: When the eye is adapted to daylight, the diagram is divided into reddish and greenish hues by a line that runs from approximately 475 to $575 \mathrm{~nm}$; and into bluish and yellowish hues that runs from approximately $520 \mathrm{~nm}$ to D65 and then nearly horizontally. b. A magnified section of the MacLeod-Boynton diagram showing the five lines along which measurements were made in Experiment 1. Along each line, chromatic discrimination was measured at 9 referent chromaticities and the paired points directly represent the separation of the discriminanda needed to support $79.4 \%$ correct discrimination in the case of one observer (S2). Inset: the target array. 


\section{METHODS.}

\section{A. Apparatus.}

The experiments were performed in St. Petersburg and in Cambridge using similar apparatus and similar programs. In both laboratories the stimuli were presented on a Mitsubishi Diamond Pro 2070 monitor. The monitor in St. Petersburg was driven at a resolution of $1280 \times 960$ pixels with a refresh rate of $92 \mathrm{~Hz}$; in Cambridge the corresponding values were $1024 \times 768$ pixels and 100 Hz. Similar graphics controllers were used to display the stimuli (Cambridge Research Systems ViSaGe in St. Petersburg and VSG2/3 in Cambridge, giving output resolutions of 14 and 15 bits per gun respectively). Screens were linearized with an OptiCal photodiode in St. Petersburg and a ColorCal in Cambridge. The spectral power distribution for each gun at maximal output was measured with a JETI spectroradiometer.

\section{B. Stimuli.}

A steady background metameric to CIE illuminant D65 and of luminance $10 \mathrm{~cd} / \mathrm{m} 2$ was always present. For discrimination measurements, the stimulus array consisted of a circular foveal field (diameter $2 \mathrm{deg}$ ), divided into four quadrants, one of which randomly differed in chromaticity from the other three (See inset Fig. 1(b)). The average luminance of the quadrants was $13 \mathrm{~cd} / \mathrm{m} 2$, but a luminance jitter of $\pm 1 \%$ was added to each quadrant randomly and independently. The individual quadrants were separated by thin (1 pixel) lines of the same chromaticity and luminance as the background, since a small gap is known to improve color discrimination [36].

The stimulus array was presented for $150 \mathrm{~ms}$. This brief duration was chosen to minimize the disturbance to the observer's state of adaptation: Self-adaptation to a prolonged test stimulus - as would have been possible in some earlier studies - would blur any attempt to show enhanced discrimination at a specific locus in the chromaticity diagram, since the underlying chromatic channels would begin their adjustment towards a new equilibrium point (For discussion, see [35]).

For phenomenological measurements of unique red, the stimulus was a uniform disk of the same diameter and duration as used for the discrimination measurements. The luminance of the disk was on average $13 \mathrm{~cd} / \mathrm{m} 2$, but the same $\pm 1 \%$ jitter was introduced as for the discrimination measurements.

For both types of measurement, viewing was binocular from a distance of $570 \mathrm{~mm}$. Fixation was guided by a diamond array of four dark dots, arranged symmetrically around the position of the stimulus field and present continuously.

We express the chromaticities of our stimuli in a version of the MacLeod-Boynton diagram, constructed from the cone sensitivities of DeMarco, Pokorny and Smith [37]. The scaling of the ordinate of the MacLeod-Boynton diagram is arbitrary: It was originally chosen so that $\mathrm{S} /(\mathrm{L}+\mathrm{M})$ is 1.0 at $400 \mathrm{~nm}$. We have rescaled the ordinate of our diagram so that a line of unique blues and unique yellows runs with a slope of $-45^{\circ}$ (Figure 1(b)). The scaling factor we adopt for the ordinate is 1.64 relative to the original diagram. Thus the coordinates of D65 change from $0.6562,0.0167$ to $0.6562,0.0273$.

\section{C. Procedure.}

In separate, but interleaved, experimental sessions we made two types of measurements: Performance measurements of chromatic discrimination in a four-alternative spatial forced-choice task, and phenomenological estimates of the chromaticities that appeared unique red, that is, neither bluish nor yellowish. At the beginning of all experimental sessions, observers adapted to the neutral background field for 1 min before measurements started.

In Experiment 1, threshold measurements were made along five lines running parallel to the locus of unique yellows. These lines intersected the horizontal line through D65 at $\mathrm{L} /(\mathrm{L}+\mathrm{M})$ values of 0.6552 (D65), 0.675, 0.695, 0.715 and 0.735 (see Fig. 1(b)). The observer was asked on each trial to indicate which of the stimulus quadrants differed from the other three. Responses were gathered via a four-button response box (CT3, Cambridge Research Systems). Auditory feedback was given. In a given experimental session, we made measurements at each of nine 'referent' chromaticities along one of the five lines of Fig. 1b, testing the nine chromaticities in random order. The referent chromaticity was never itself presented: The discriminanda lay on the same line in chromaticity space and straddled the referent chromaticity. The target quadrant could differ from the distractor quadrants randomly in either direction, i.e., as an increment or a decrement in S-cone excitation relative to the referent. The chromatic separation of the discriminanda was increased or decreased symmetrically around the referent according to the observer's accuracy. The staircase procedure tracked $79.4 \%$ correct [38]. The referent and test chromaticities were expressed in terms of the abscissa of the MacLeod-Boynton diagram (i.e. the $\mathrm{L} /(\mathrm{L}+\mathrm{M})$ or $l$ coordinate). At any one point on the staircase, one of the discriminanda had an $l$ coordinate $l_{t 1}$, and the other had an $l$ coordinate $l_{t 2}$, where $l_{t 1}$ was equivalent to the reference coordinate $l_{r}$ multiplied by a factor $\boldsymbol{a}$ and $l_{t 2}$ was equivalent to $l_{r}$ divided by $\boldsymbol{a}$, where $\boldsymbol{a}$ is always $>1.0$. After three correct responses, the value $(\boldsymbol{a}-1)$ was reduced by $10 \%$, and after each incorrect response it was increased by $10 \%$. The staircase terminated after 15 reversals, the last 10 reversal points being averaged to give the threshold. There were 6 sets of experimental runs, the first set being treated as practice and not included in the analysis. Thus any given threshold for a given subject is based on 5 independent repetitions.

In six additional experimental sessions, interleaved with the discrimination measurements, we estimated the chromaticity of the observer's unique red - the subjective transition point between bluish and yellowish hues. In individual blocks of trials within one experimental session, the chromaticity of the uniform target disk was varied along one of seven $-45^{\circ}$ lines in our MacLeod-Boynton space, and the observer was asked to indicate by pushbuttons whether it appeared too yellowish or too bluish to be unique red. No feedback was given. To avoid sequential effects in these phenomenological measurements, four randomly-interleaved staircases were used to estimate the transition point between reddish and greenish hues, two staircases starting on each side of the expected match [39] - a procedure that has been estimated to give a stimulus sequence as random as that of the Method of Constant Stimuli [40, 41] while avoiding the central tendency produced by a fixed set of constants [42]. Each staircase terminated after 15 reversals. The last 10 reversals of each of the 4 staircases were pooled to give an estimate of the unique hue for a given line. In any one experimental session, the perceptual transition points were estimated for different $-45^{\circ}$ lines, in a different random order in different sessions.

\section{D. Observers.}

There were four observers (S1-S4). S1 and S2 were the authors. The other observers were highly practiced, but were naïve as to the purpose of the present experiment. All observers except JDM were female and all observers had normal color vision as tested by the Cambridge Colour Test $[43,44]$. S2 and S3 were tested in St Petersburg, S1 and S4 in Cambridge. 


\section{EXPERIMENT 1: RESULTS AND DISCUSSION.}

An example of the discrimination results for Experiment 1 is shown in a very direct way for one observer (S2) in Figure 1b: The pairs of data points show the separation of chromaticities needed to allow the observer to achieve the criterion level of discrimination performance (79.4\% correct). Notice that the paired points vary in separation along each line and that the separations are smallest - discrimination is optimal - for chromaticities on or just below the line that runs horizontally through D65.

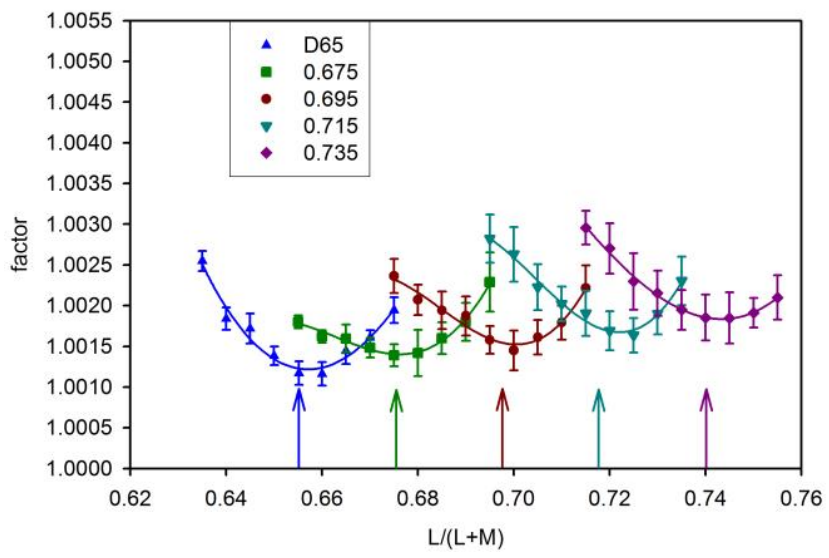

Figure 2. Average threshold measurements for Experiment 1 . The abscissa represents the $\mathrm{L} /(\mathrm{L}+\mathrm{M})$ coordinate of the chromaticity at which the measurement was made. The ordinate represents a measure of discrimination: the factor by which the target and distractor quadrants needed to differ from the referent chromaticity in order to sustain $79.4 \%$ correct performance. The error bars represent \pm 1 SEM. Each dataset in the figure, represented by a different symbol,

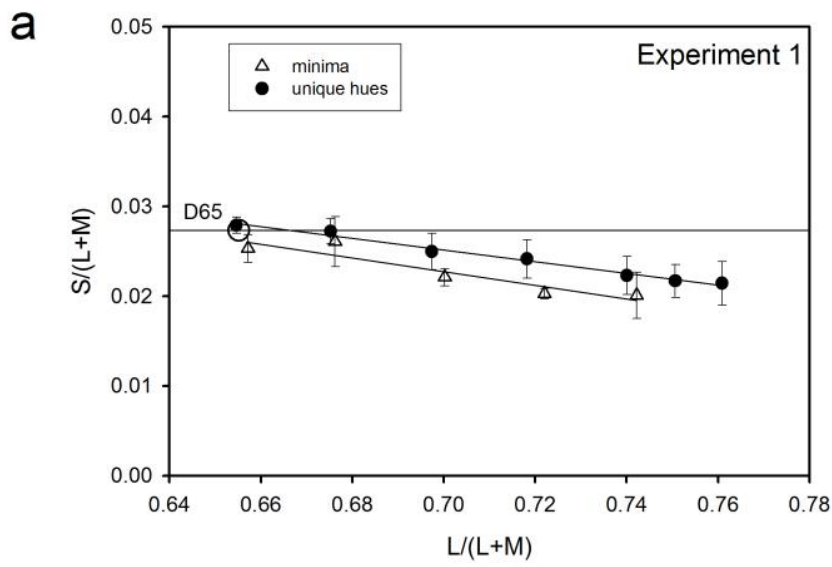

corresponds to one of the lines in Figure 1b. The curves fitted to the datasets are inverted third-order polynomials and have no theoretical significance. The vertical arrows indicate the average $\mathrm{L} /(\mathrm{L}+\mathrm{M})$ coordinate of unique red, obtained from observers' settings in experimental runs interleaved with the threshold measurements. Note that the minimal thresholds always coincide approximately with the subjective boundary between bluish and yellowish hues.

Figure 2 shows average discrimination thresholds for all observers. Each dataset corresponds to thresholds for one of the five lines in Figure 1b. The abscissa of the graph represents the $L /(L+M)$ coordinate of the referent chromaticity at which a given measurement was made and the ordinate represents the factor by which the positive and negative discriminanda differ at threshold from the referent chromaticity (see Methods). All the five sets of data exhibit a clear minimum.

Below each of the five sets of threshold data in Figure 2 we have plotted an arrow that indicates the measured chromaticity of unique red for that line. There is a fair agreement between the chromaticity of the unique hue and the position of the minimal threshold. In other words, in this dataset, the subjective category boundary between yellowish and bluish hues does approximately coincide with a chromaticity region of enhanced discrimination.

The relationship between phenomenological and performance measures can be judged more systematically in Figure 3a, where the chromaticities of equilibrium hues and of minimal thresholds are plotted in a portion of the MacLeod-Boynton diagram. The locus of unique hues is shown by the filled circles. The locus does not run quite horizontally from the white point (D65) but descends to lower $S$ values as $\mathrm{L} /(\mathrm{L}+\mathrm{M})$ increases. In this characteristic, our average results resemble those derived for Munsell samples by Valberg [45] and those obtained in CRT-based measurements by Wuerger, Atkinson and Cropper [9] - although there were individual differences among our observers similar to those recorded by others [7].

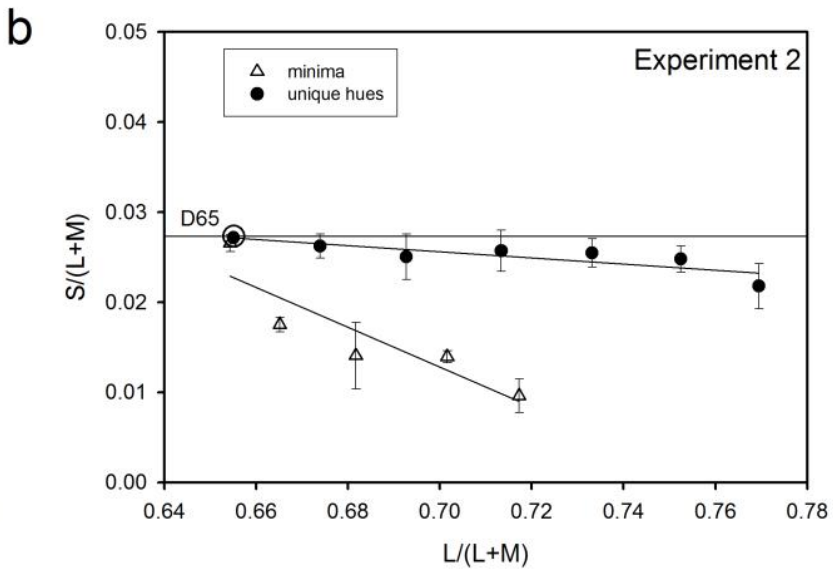

Figure 3. a. A magnified section of the MacLeod-Boynton chromaticity diagram showing summary results from Experiment 1 , where measurements were made along lines parallel to the locus of unique blue and unique yellow. The solid circles show the average settings for unique red and the open triangles show the chromaticities at which discrimination thresholds are minimal. To obtain the latter estimates, inverse polynomials were independently fitted to the thresholds for each observer. The data points are the average of these estimates; the error bars represent \pm 1 SEM and correspond to between-observer variance. Note that the locus of unique red diverges systematically from a strictly horizontal line through the chromaticity of Illuminant D65, and that the locus of minimal thresholds runs close to, and parallel with, the locus of equilibrium hues.

b. Corresponding results from Experiment 2, where measurements were made along lines orthogonal to the locus of unique blues and unique yellows. Note that again the locus of unique red descends below a horizontal line through the chromaticity of D65, but now the locus of minimal thresholds diverges strongly from the locus of equilibrium hues.

The open triangles in Figure 3a represent the average positions of the minimal thresholds, estimated by fitting inverse polynomials to the datasets for each observer. There is no significant difference in slope between the regression lines fitted to the phenomenological and discrimination data $(\mathrm{t}=0.8, d f=8, p=0.446)$, but there is a significant offset between the two sets of points $(\mathrm{t}=-5.46, d f=9, p=$ 
0.0004). The small but significant offset may be accounted for by subjective biases in the phenomenological judgments. Whereas the threshold measurements required discrimination of individual quadrants, the phenomenological judgments were based on the appearance of the entire 2-deg disk: There was typically a range of chromaticities where observers reported the disks to appear neither uniformly orange nor uniformly salmon.

The parallelism between the phenomenological and performance data in Figure 3a is striking. These results would be compatible with the hypothesis that the minimal thresholds occur at or near the equilibrium point of a neural channel that signals blueness and yellowness (or alternatively, the equilibrium points of paired channels that signal blueness and yellowness respectively). An analogous conclusion could be drawn from our earlier measurements at the locus of unique blues and yellows, i.e. at the subjective boundary between reddish and greenish hues [20,34]; but at the locus of unique green the phenomenological equilibria and the optimal discrimination did not coincide [35]. In Experiment 2, when the locus of unique red is traversed in a different direction, we find a result very different from that of Experiment 1.

\section{EXPERIMENT 2: RESULTS AND DISCUSSION.}

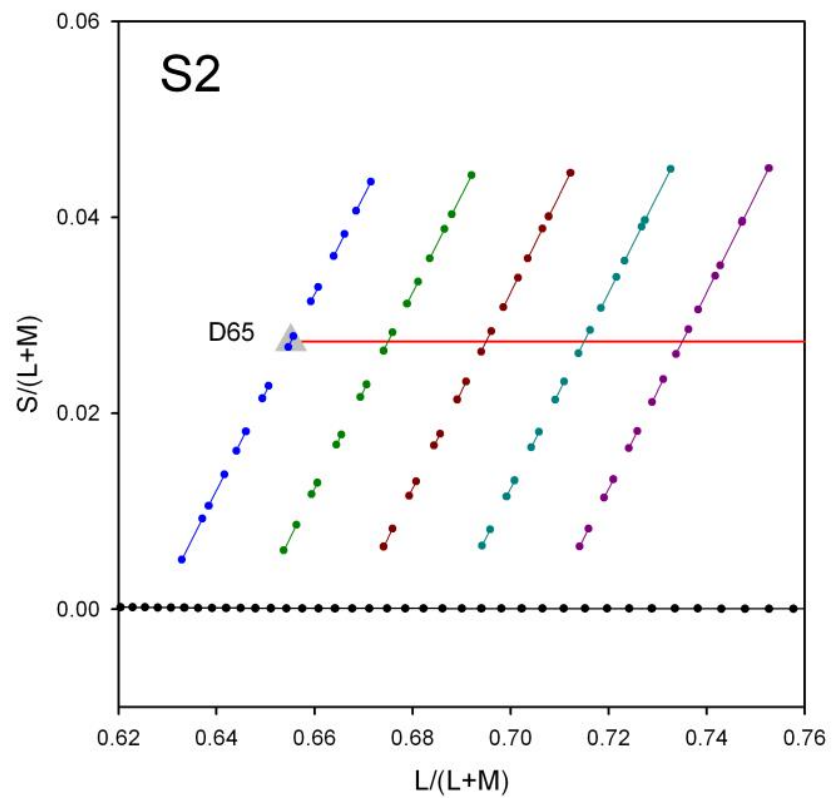

Figure 4. A magnified section of the MacLeod-Boynton diagram showing the five lines along which measurements were made in Experiment 2. Along each line, chromatic discrimination was measured at 8 referent chromaticities and the paired points directly represent the separation of the discriminanda needed to support $79.4 \%$ correct discrimination in the case of one observer (S2).

In the second experiment, we made threshold measurements as in Experiment 1 but along lines that were oriented at $+45^{\circ}$ in our scaled MacLeod-Boynton space and thus cut through the locus of unique red from a different angle (see Fig. 4). These lines are approximately orthogonal to the locus of unique blues and unique yellows. Eight referent chromaticities were tested along each of a set of five lines that intersected the horizontal line through $\mathrm{D} 65$ at $\mathrm{L} /(\mathrm{L}+\mathrm{M})$ values 0.6552(D65), 0.675, 0.695, 0.715, 0.735 (Fig 4). As before, we also obtained estimates of unique red in independent experimental sessions, interleaved with the sessions in which thresholds were measured, but now the target disk was varied along lines orthogonal to the locus of unique blues and yellows. All other experimental arrangements were as in Experiment 1. There were four observers, who had each earlier served in Experiment 1.

The primary difference between Experiments 1 and 2 is the phase in which the S-cone modulation was combined with the modulation of the L/M signal: in Experiment 1, increments in the S-cone signal were in phase with increments in the M-cone signal, whereas in Experiment 2, they were in phase with increments in the L-cone signal.

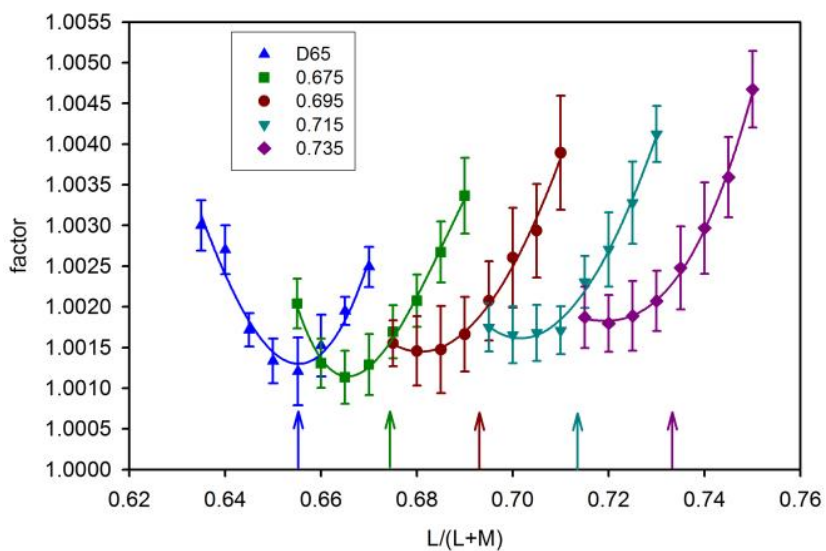

Figure 5. Average threshold measurements for Experiment 2. The abscissa represents the $\mathrm{L} /(\mathrm{L}+\mathrm{M})$ coordinate of the chromaticity at which the measurement was made. The ordinate represents a measure of discrimination: the factor by which the target and distractor quadrants needed to differ from the referent chromaticity in order to sustain $79.4 \%$ correct performance. The error bars represent \pm 1 SEM. Each dataset in the figure, represented by a different symbol, corresponds to one of the lines in Fig 4 . The curves fitted to the datasets are inverted third-order polynomials and have no theoretical significance. The vertical arrows indicate the $\mathrm{L} /(\mathrm{L}+\mathrm{M})$ coordinate of unique red, obtained from observers' settings in experimental runs interleaved with the threshold measurements. For all datasets except for the line passing through D65, there is a discrepancy between the chromaticity of unique red and the chromaticity at which discrimination is optimal.

Figures 4 and 5 show the results of this second experiment. In Fig. 4 the yoked data points represent directly the separation of chromaticities needed for one observer (S2) to discriminate them at a performance level of 79.4\%. In Fig. 5 we give average thresholds for the five sets of data. The abscissa shows the $\mathrm{L} /(\mathrm{L}+\mathrm{M})$ coordinate at which chromatic discrimination was measured; and the ordinate shows the factor by which the positive and negative discriminanda differed from the referent chromaticity at threshold. Different datasets correspond to different lines in Fig. 4. In the case of the line passing through D65 - the white adapting point - there is a clear minimum at the white point (when we may expect all neural channels to be in their equilibrium state. For lines at higher values of $\mathrm{L} /(\mathrm{L}+\mathrm{M})$ the minimum moves to lower values of $\mathrm{S} /(\mathrm{L}+\mathrm{M})$ and approaches the limit of the gamut set by the red and green guns of our monitor.

The vertical arrows in Fig. 5 represent for each line the concurrently measured estimate of unique red (or white in the case of the line passing through D65) - the boundary between bluish and yellowish hues. At the white point, as is almost invariably found [e.g. $26,46,47]$, the minimal threshold falls close to the neutral hue, but as the $\mathrm{L} /(\mathrm{L}+\mathrm{M})$ coordinates of the datasets increase, the phenomenal 
equilibrium point clearly lies at $\mathrm{L} /(\mathrm{L}+\mathrm{M})$ coordinates higher than that of the minimal threshold.

Fig. $3 \mathrm{~b}$ allows a systematic comparison of the phenomenological equilibria (solid circles) and the minimal thresholds (open triangles) obtained in Experiment 2. As in Experiment 1, the locus of unique red is not quite horizontal, but descends to lower values of $\mathrm{S} /(\mathrm{L}+\mathrm{M})$ as $\mathrm{L} /(\mathrm{L}+\mathrm{M})$ increases. However, the discrimination and the phenomenological measures no longer show the parallelism seen in Experiment 1: The locus of minimal thresholds descends much more steeply and there is a significant difference in the slopes of the regression lines ( $\mathrm{t}=3.97, d f=8, p=0.004)$.

\section{GENERAL DISCUSSION.}

A unique hue by definition represents a category boundary, either between reddish and greenish hues or (as studied here) between bluish and yellowish hues. If a particular chromaticity in a particular state of adaptation has a unique hue, then we might expect that it remains a unique hue from whatever direction it is approached (provided psychophysical precautions are taken to avoid sequential biases or self-adaptation to the test flash). Our present two experiments were carried out many months apart, but the locus of unique red remains relatively stable for our observers under the two conditions of estimation (Figs 3(a) and 3(b)).

But should we expect discrimination always to be enhanced near the unique hue, independently of the direction in which the hue boundary is crossed? When color categories are explained in terms of central processes, a mechanistic explanation is seldom offered for any enhancement of discrimination near the boundary; and so it is not easy to envisage how the discrimination would be affected by the direction in which the measurement is made.

If, however, the question is considered from the perspective of visual science, it is plain that enhancement might sometimes be expected and sometimes not. It is exceedingly unlikely that signals from the same underlying neural channel would be used for discrimination along all directions through a single point in chromaticity space [see e.g. 22, 23, 29]. For some directions, indeed, the channel that determines the perceptual hue boundary may also be the channel that is used for discrimination; but this is unlikely always to be the case.

When making our earlier measurements orthogonal to the line of unique blues and yellows, we found that optimal discrimination coincided with the locus of phenomenological equilibrium - the boundary between reddish and greenish colors [20,34]. This was also the case when we traversed the same yellow-blue line along a horizontal trajectory in the MacLeod-Boynton diagram, i.e. along a trajectory that varies $\mathrm{L} /(\mathrm{L}+\mathrm{M})$ but holds constant the excitation of short-wave cones [48]. On the other hand, we found no indication of enhanced discrimination at the locus of unique green when we made measurements orthogonal to the locus; rather the minimal thresholds always coincided with the $\mathrm{L} /(\mathrm{L}+\mathrm{M})$ coordinate of the adapting field, as would be expected if discrimination depended on a traditional midget ganglion cell pathway [35].

In the present study we have measured discrimination in two different directions across the locus of unique red. The discrimination measurements differed primarily in the sign with which S-cone modulations were combined with modulations of the L/M signal: In Experiment 1 increments and decrements in $\mathrm{S}$ are paired with increments and decrements in the M-cone signal, whereas in Experiment 2, increments and decrements in $S$ are paired with increments and decrements in the L-cone signal. When measurements are made in the first direction, the minimal thresholds do approximately coincide with the phenomenal hue boundary (and both measures might depend on the same neural channel, one in which $\mathrm{S}$ and $\mathrm{M}$ signals are synergistic and are opposed to L signals); but in the orthogonal direction, the threshold minima systematically differ from the boundaries of the perceptual categories.

In sum, our results from these, and from earlier experiments on the other unique hues, could be explained if the channel that determines thresholds is sometimes the same as the channel that determines the category boundary - and sometimes is not. If a given point in the chromaticity diagram is a unique hue, it remains (in principle) a unique hue whatever the direction in which it is measured. On the other hand, it is very unlikely that discrimination thresholds will depend on the same underlying neural channel when measurements are made through the same chromaticity point but in many different directions.

Our approach of measuring thresholds along transects of a chromaticity diagram is in the tradition of 'Wright's dashes' [49] - in contrast to that of 'MacAdam's ellipses' [50]. When discrimination ellipses have been measured, the tradition has been to measure them either at arbitrary positions in color space or to center them on cardinal axes [23]. Our results suggest that it may be analytically useful in a future experiment to center discrimination ellipses on the loci of unique hues.

\section{References}

1. Rushton, W.A.H., Pigments and signals in colour vision. Journal of Physiology, 1972. 220: p. 1-31P.

2. MacLeod, D.I.A. and R.M. Boynton, Chromaticity diagram showing cone excitation by stimuli of equal luminance. Journal of the Optical Society of America, 1979. 69: p. 1183-1186.

3. Smith, V.C. and J. Pokorny, The design and use of a cone chromaticity space: a tutorial. Color Research and Application, 1996. 21: p. 375-382.

4. S.A. Burns, A. E. Elsner, J. Pokorny and V. C. Smith (1984). "The Abney Effect: chromaticity coordinates of unique and other constant hues." Vision Research 24: 479-489.

5. Webster, M.A. and J.D. Mollon, The influence of contrast adaptation on color appearance. Vision Research, 1994. 34: p. 1993-2020.

6. Nerger, J.L., V.J. Volbrecht, and C.J. Ayde, Unique hue judgements as a function of test size in the fovea and at 20-deg temporal eccentricity. Journal of the Optical Society of America, 1995. 12A: p. 1225-1232.

7. M.A. Webster, E. Miyahara, G. Malkoc and V. E. Raker (2000). "Variations in normal color vision. II. Unique hues." Journal of the Optical Society of America 17A: 1545-1555.

8. Mollon, J.D. and G. Jordan, On the nature of unique hues, in John Dalton's Colour Vision Legacy, C. Dickinson, I. Murray, and D. Carden, Editors. 1997, Taylor and Francis: London. p. 381-392.

9. Wuerger, S.M., P. Atkinson, and S. Cropper, The cone inputs to the unique-hue mechanisms. Vision Res, 2005. 45(25-26): p. 3210-23.

10. A.M. Liberman, K. S. Harris, H. S. Hoffman and B. C. Griffith (1957). "The discrimination of speech sounds within and across phoneme boundaries." Journal of Experimental Psychology 54(5): 358-368.

11. A.M. Liberman, H. Lane, K. S. Harris and J. A. Kinney (1961). "Discrimination of Relative Onset-Time of Components of Certain Speech and Nonspeech 
Patterns." Journal of Experimental Psychology 61(5): 379-388. Bornstein, M.H. and N.O. Korda, Discrimination and matching within and between hues measured by reaction times: some implications for categorical perception and levels of information processing. Psychological Research, 1984. 46: p. 207-222.

13. Goldstone, R.L. and A.T. Hendrickson, Categorical perception. Wiley Interdisciplinary ReviewsCognitive Science, 2010. 1(1): p. 69-78.

14. Webster, M.A. and P. Kay, Color categories and color appearance. Cognition, 2012. 122(3): p. 375-392.

$15 . \quad$ R.J. Bachy, Dias, D. Alleysson and V. Bonnardel (2012). "Hue discrimination, unique hues and naming." Journal of the Optical Society of America aOptics Image Science and Vision 29(2): A60-A68.

16. Witzel, C. and K.R. Gegenfurtner, Categorical sensitivity to color differences. Journal of Vision, 2013. 13(7,1): p. 1-33.

17. Winawer, J., Witthoft, N., Frank, M. C., Wu, L., Wade, A. R., Boroditsky, L., Russian blues reveal effects of language on color discrimination. Proceedings of the National Academy of Sciences of the United States of America, 2007. 104: p. 7780-7785.

18. Roberson, D., J.R. Hanley, and H. Pak, Thresholds for color discrimination in English and Korean speakers. Cognition, 2009. 112: p. 482-487.

19. Paramei, G.V., Russian 'blues'. Controversies of basicness, in Anthropology of Color, R.E. MacLaury, G.V. Paramei, and D. Dedrick, Editors. 2007, John Benjamins Publishing Co.: Amsterdam. p. 75-106.

20. Danilova, M.V. and J.D. Mollon, Parafoveal color discrimination: a chromaticity locus of enhanced discrimination. Journal of Vision, 2010. 10(1).

21. Stiles, W.S., A modified Helmholtz line-element in brightness-colour space. Proceedings of the Physical Society, 1946. 58: p. 41-65.

22. Le Grand, Y., Les seuils différentiels de couleurs dans la théorie de Young. Revue d'Optique théor. exp., 1949. 28: p. 261-278.

23. Boynton, R.M. and N. Kambe, Chromatic Difference Steps of Moderate Size Measured Along Theoretically Critical Axes. Color Research and Application, 1980. 5(1): p. 13-23.

24. Yeh, T., J. Pokorny, and V.C. Smith, Chromatic Discrimination with Variation in Chromaticity and Luminance - Data and Theory. Vision Research, 1993. 33(13): p. 1835-1845.

25. Mollon, J.D., O. Estévez, and C.R. Cavonius, The two subsystems of colour vision and their rôles in wavelength discrimination, in Vision: coding and efficiency, C. Blakemore, Editor. 1990, Cambridge University Press: Cambridge. p. 119-131.

26. Krauskopf, J. and K. Gegenfurtner, Color discrimination and adaptation. Vision Research, 1992. 32(11): p. 2165-75.

27. Cole, G.R., T. Hine, and W. Mcilhagga, Detection Mechanisms in L-Cone, M-Cone, and S-Cone Contrast Space. Journal of the Optical Society of America aOptics Image Science and Vision, 1993. 10(1): p. 3851.

28. C.F. Stromeyer, A. Chaparro, C. Rodriguez, D. Chen, E. $\mathrm{Hu}$ and R. E. Kronauer (1998). "Short-wave cone signal in the red-green detection mechanism." Vision Research 38(6): 813-826.

29. Eskew, R.T., Jr., Higher order color mechanisms: a critical review. Vision Res, 2009. 49(22): p. 2686704.

30. Byzov, A.L. and L.P. Kusnezova, On the mechanisms of visual adaptation. Vision Research Supplement, 1971. 3: p. 51-63.

31. De Valois, R.L., I. Abramov, and W.R. Mead, Single cell analysis of wavelength discrimination at the lateral geniculate nucleus in the macaque. Journal of Neurophysiology, 1967. 30: p. 415-433.

32. Derrington, A.M., J. Krauskopf, and P. Lennie, Chromatic mechanisms in lateral geniculate nucleus of macaque. Journal of Physiology, 1984. 357: p. 241-65.

33. Lee, B.B. and D.M. Dacey, Structure and function in primate retina, in Colour Vision Deficiencies XIII, C.R. Cavonius, Editor. 1997. p. 107-117.

34. Danilova, M.V. and J.D. Mollon, Foveal color perception: Minimal thresholds at a boundary between perceptual categories. Vision Research, 2012. 62: p. 162-172.

35. Danilova, M.V. and J.D. Mollon, Is discrimination enhanced at the boundaries of perceptual categories? A negative case. Proceedings of the Royal Society BBiological Sciences, 2014. 281(1785).

36. Boynton, R.M., M.M. Hayhoe, and D.I.A. MacLeod, The gap effect: chromatic and achromatic visual discrimination as affected by field separation. Optica Acta, 1977. 24: p. 159-177.

37. DeMarco, P., J. Pokorny, and V.C. Smith, FullSpectrum Cone Sensitivity Functions for $X$ -

Chromosome-Linked Anomalous Trichromats. Journal of the Optical Society of America A-Optics Image Science and Vision, 1992. 9(9): p. 1465-1476.

38. Wetherill, G.B. and H. Levitt, Sequential estimation of points on a psychometric function. British Journal of Mathematical and Statistical Psychology, 1965. 18: p. 1-10.

39. Jordan, G. and J.D. Mollon, Rayleigh matches and unique green. Vision Res, 1995. 35(5): p. 613-20.

40. Sternberg, S., R.L. Knoll, and B.A. Gates, Prior entry reexamined: effect of attentional bias on order perception, in Annual Meeting of the Psychonomic Society. 1971: St. Louis.

41. Sternberg, S., R.L. Knoll, and P. Zukofsky, Timing by skilled musicians: perception, production, and imitation of time ratios, in The Psychology of Music, D. Deutsch, Editor. 1982, Academic Press: New York.

42. Poulton, E.C., Models for Biases in Judging Sensory Magnitude. Psychological Bulletin, 1979. 86(4): p. 777-803.

43. Mollon, J.D. and J.P. Reffin, A computer-controlled colour vision test that combines the principles of Chibret and of Stilling. Journal of Physiology, 1989. 414: p. 5P.

44. Regan, B.C., J.P. Reffin, and J.D. Mollon, Luminance noise and the rapid determination of discrimination ellipses in colour deficiency. Vision Research, 1994. 34: p. 1279-1299. 
45. Valberg, A., Unique hues: an old problem for a new generation. Vision Research, 2001. 41(13): p. 16451657.

46. Miyahara, E., V.C. Smith, and J. Pokorny, How Surrounds Affect Chromaticity Discrimination. Journal of the Optical Society of America a-Optics Image Science and Vision, 1993. 10(4): p. 545-553.

47. Rautian, G.N. and V.P. Solov'eva, Vlijanie svetlogo okrugenija na ostrotu cvetorazlochenija. Doklady Academii Nauk SSSR, 1954. 95: p. 513-515.

48. Danilova, M.V. and J.D. Mollon, Cardinal axes are not independent in color discrimination. Journal of the Optical Society of America a-Optics Image Science and Vision, 2012. 29(2): p. A157-A164.

49. Wright, W.D., The sensitivity of the eye to small colour differences. Proceedings of the Physical Society, 1941. 53: p. 93-112.

50. MacAdam, D.L., Visual sensitivities to color differences in daylight. Journal of the Optical Society of America, 1942. 32: p. 247-281.

51. Wyszecki, G. and W.S. Stiles, Color Science. 2nd ed. 1982, New York: Wiley. 